\title{
Exploring Nursing Students Perception on High-fidelity Practices: A Phenomenological Study
}

\author{
Fatma Baddar $^{1,2}$, Norah Abdulrahman ${ }^{3}$, Huda Mhawish ${ }^{4}$, Olfat Atia Salem ${ }^{1,5}$ \\ ${ }^{1}$ Department of nursing administration and education, College of Nursing, King Saud University, Riyadh, Kingdom of Saudi Arabia \\ ${ }^{2}$ Nursing Administration Department, Faculty of Nursing, Alexandria, Egypt \\ ${ }^{3}$ Department of Nursing Administration and Education, College of Faculty of Applied Medical Sciences in Dawadmi, Shaqra University, \\ Riyadh, Kingdom of Saudi Arabia \\ ${ }^{4}$ Administrative Nurse Manager, Critical Care Department, King Saud Medical City, Riyadh, Saudi Arabia \\ ${ }^{5}$ Nursing Administration Department, Faculty of Nursing, Menoufia University, Menoufia, Egypt
}

Email address:

Fbadar@ksu.edu.sa(F.Baddar),nura_a@su.edu.sa(N.Abdulrahman),huda.mhawish@gmail.com(H. Mhawish)

\section{To cite this article:}

Fatma Baddar, Norah Abdulrahman, Huda Mhawish, Olfat Atia Salem. Exploring Nursing Students Perception on High-fidelity Practices: A Phenomenological Study. Clinical Medicine Research. Vol. 8, No. 4, 2019, pp. 69-76. doi: 10.11648/j.cmr.20190804.11

Received: July 25, 2019; Accepted: August 20, 2019; Published: September 4, 2019

\begin{abstract}
High-fidelity simulation (HFS) is an instructive/learning technique progressively utilized by nursing schools, where students experience mimicked clinical encounters creating specialized and non-specialized abilities. The goal of this trial is to understand the perceptions of students of the bachelor's degree in Nursing with respect to their cooperation in reproduced clinical encounters with HFS. A qualitative investigation of phenomenological approach was undertaken as a design of this study. A convenient sample of 8 undergraduate nursing students involved in the investigation were interviewed. Later, a fundamental structure of the nurses' perception was developed which reflected the real clinical situations and other scenarios on exposure to HFS. The result of the study founded that the students were able to develop confidence based on segments such as enjoying the HFS settings, association with peers in the simulation, and skill development. The students appear to be happy with their experience of HFS. Negative perception is poisonous in any learning process. It may result from a factor beyond the control of the student such as shortage of equipment and cost. Lack of opportunity will undermine the senses of reality, experience, and chance to put their skills in practice. Moreover, the effects of such negative perception would make students frustrated and unable to learn from their errors. The study recognized a distinctive impression of students after encountering reasonable situations in a controlled domain, its significance to the foundation of relations and common development and its importance for their future as students and future experts.
\end{abstract}

Keywords: High-fidelity Simulation, Simulation, Nursing Students, Students Simulation

\section{Introduction}

This Simulation is a strategy to supplant or enhance genuine encounters with guidance. It is frequently, provides immersive in nature and lets the student inspire or feel the generous parts of reality in a completely intuitive manner. Simulation in the territory of pharmaceutical and nursing has turned into an imperative piece of the instruction for students and rehearsing medical services suppliers. High-fidelity simulation (HFS) is progressively a mainstream source in nursing [1].

Available sources have uncovered knowledge that, on the possibility of High-faithfulness proliferation, with the likelihood to enhance scholarly, familiar, and free aptitudes, can empower students to make clinical reasoning. In such way, a gathering of masters broke down third-year students' (three hundred students) observations about the use of high-commitment proliferation within the current clinical course. Students' transient notion of perplexity was deciphered as they separated, gathered, and made an interpretation of signs to respond to quick changes in the imitated patient's clinical condition [2].

Clinical experience is seen as a wellspring of fear and uneasiness for nursing students [3]. Researchers found that the majority of techniques do not reflect clinical reality or are 
excessively costly. A present report had stated that (HFS) has been utilized as a part of nursing guideline for more than 20 years. They attempted to discuss the upsides of the use of high-consistency simulation in nursing schools [4]. HFS has been planned as a part of the clinical examination course of a fundamental year in the baccalaureate nursing program in one of the nursing schools in Macau.

Thus, the researchers concluded that HFS practices as a component of the clinical examination course in such nursing school is an amazing decision and the first of its kind in Macau. These HFS practices as opposed to certifiable clinical positions were esteemed by nursing students. In addition, it fundamentally added to the inventive limit in students' view. The examination incorporated that in the midst of HFS working out, nursing educators should consider the misguided judgment of HFS activities of students who hated test framework's and the reiterated learning procedure. However, the group failed to consider the HFS usage over expanded time allotments for checking better outcomes among the students [4]. This examination appeared to strengthen a past report by Haraldseid, Friberg and Aase (2015).

These pros have put revolve around the way that specialist of clinical aptitudes learning is required to twist up the readied orderly. They concentrated on it as a result of limited open entryways for clinical capacities getting ready in clinical practice. Student planning at clinical aptitudes inquiry about offices (CSLs) is a central part of the nursing direction. The examination highlighted that a sociocultural and perspective learning is masterminded in a circumstance. Everything considered, they inspected those creating understudy partners, fast introduction of advancements, based indicating procedures, and a move from education to a learning-center preparing for all effects on the students. The examination concluded that movements impact CSLs and drive nursing assets to acclimate to the changing learning condition [5].

Afterward, Ha (2016) announced that the OSCE (Observed organized clinical examination) is a powerful instructive strategy to assess the clinical skills of students. In this way, they distinguished the subjective states of mind of undergrad nursing students to a $\mathrm{C}$-SOSCE (educational modules for simulation based OSCE) [6].

Baptista et al. (2016) have featured the utilization of reproduction to recreate the experience of social insurance settings, and its utilization as a system in the instructing of attendants has developed at a noteworthy rate. They express that there is minimal reason to look at the distinctions in increasing fulfillments by students with the utilization of medium and high fidelity. The study team tried to dissect and benchmark additions and fulfillments saw by nursing students, as indicated by their support in medium-and high-constancy reproduced practice.

Finally, studies have pointed out that negative perception have tremendous impact on students' learning ability. Aspects such as shortage of equipment as well as lack of opportunity will deny learners the sense of reality. Individuals are likely to lose on their ability to develop confidence and learn to appreciate their worth [7]. Manchak et al (2014) pointed out that the best office practices should call for the provision of all necessities to allow for the effective adaption and nurturing of skills [8].

Littlewood (2011) states that Simulation is becoming typical in clinical training and can also be utilized as an investigative clinical instrument in its own particular right. Utilization of HFS isn't restricted to the medical and aeronautical fields; it is developed in nonmedical associations too at an increasing rate. The methods and educational apparatuses which have advanced use in nonmedical reproductions serve as showing cases as well as roads which can help advance the development of the idea of HFS in the field of medication [9].

A group of Canadian scientists investigated the encounters in executing and utilizing HFS in the course of the recent three years, in light of a study in proficient wellbeing training. The study team observed a fast increment in the take-up of simulation-based training in Canada. They expected that the process is an extensive part of the development of strategies in situations where students can enhance their skills. Students and educators have distinguished positive learning encounters with high-devotion simulation, especially regarding complex patient care situations, multidisciplinary group situations, understudy cooperation (i.e., group based learning), and intelligent questioning [10]. This revealed insight into one longitudinal, multimethod, and coordinated trial where the analysts tried a prototypical claim to a fame office $(\mathrm{n}=184)$ and a conventional organization $(n=177)$ in practice, particular to probationers' run infringement, and probationers' treatment [8].

In another examination, Breymier and Rutherford-Hemming (2017), has underscored that HFS has turned out to be indispensable in intense care establishments, related encounters, assessment, improvement of medical attendance and competency preparing. In such manner, the International Association for Clinical Simulation and Learning has built up best practice rules and structure for different aptitudes (i.g. blood transfusion). The Institute of Medicine report has conveyed understanding security issues to the front line. The blood transfusion administration is an ability for which HFS can give a sheltered domain to instruct and assess nurture skills for blood transfusion administration forms. This examination did not consider the HFS in long term utility in the intense care organizations though. [11].

\section{Research Study Context}

Students appear to possess a poor understanding of simulated clinical circumstances when the test system answers their inquiries and imitates the responses/developments of a genuine patient.

A study was undertaken to let the students overcome their poor insight on the emerging technologies like simulation tools and to understand the level of undergraduates' concerns in the nursing college with regard to their problems when exposed to HFS clinical conditions.

In one trial utilizing HFS practice, students could turn out to be surer about their future, since they feel better arranged, 
since the training created might be connected to the genuine setting. It is vital to believe in the learning procedure since it defeats the difficulties postured in settings on complex choices. Students could build up a sentiment that HFS has expanded their certainty before clinical practice and may enhance their skill in real practice [7].

\section{Research Questions}

What is your impression when utilizing high-fidelity Practices?

Sub-questions

1. Let me think about the sentiments you created at the season of simulation.

2. Let me think about the inconveniences you encountered at the time of simulated practice.

3. What effect would this simulation approach have on your examinations?

4. What impact would this reproduction approach have on your professional?

\section{Method}

\subsection{Design and Setting}

In this study, a Qualitative phenomenological approach was taken as a sociological investigation that gathers and works to translate significant information to understand and comprehend social life through the investigation focus on spots or populaces. Subjective approach is intended to uncover and illuminate the activity or results that are regularly estimated by quantitative research [12]. The study was conducted in nursing college in Saudi Arabia The Nursing College gives quality training through projects that are intended to profit present and future Saudi wellbeing needs. Its motivation and capacity were obtained from the methods of insight and strategies of the college.

\subsection{Sample and Data Collection}

Purposive sampling was employed in this study with Open-ended questions and semi- structure interviews in depth conducted face-to-face in a private classroom in the Nursing College setting. The interviews were carried out individually until the students' experiences represent repetitive expressions and phrases, which indicate the data saturation. Each interview lasted for 30 to 50 minutes. The interview consisted of two parts The initial part of the interview consisted of socio-demographic queries (specific to age, Study year, gender, marital status, and the number of simulated clinical situations the student participated). The second part included research questions. All interviews were recorded and transcribed for later analysis. During this study, the participants were protected by obtaining their informed consent and by including a detailed explanation of the nature, purpose, and implications of the study, as well as the security and confidentiality of the data.

\subsection{Data Analysis}

The study used thematic content analysis for driving themes by employing the NVivo software program for recording the transcription [13]. Topical Content Analysis (TCA) is an expressive introduction of subjective information. In this approach, interview transcript forms are gathered from the study participants. While recorded information go with textural information, this depiction of TCA is constrained to textural information [14]. The investigation utilized topical substance examination for driving subjects by utilizing the NVivo program for recording the interpretation. The investigation additionally modified topics to ensure the dependability of any examination.

\subsection{Ethical Considerations}

The study obtained approval from the Ethics Committee of the Research Unit and administration at the university. The participation of the students was voluntary. The recordings of the findings from the interviews were deleted after being analyzed. To ensure confidentiality, participants were not identified throughout the study. The students were informed that they can withdraw from the interview or the complete investigation prior to data analysis

\section{Results}

The demographic information of the nurse's students in college of nursing. All 8 participants were female students. Moreover $6(75 \%)$ respondents were single and $2(25 \%)$ students were married. Of the total 8 nurses student, $2(25 \%)$ from level 5 and same percentage also from level 6 , and also 3 (37.5\%) from level 7. Furthermore, $1(12.5 \%)$ participant is from level 8 , no one from level 4 , and $8(100 \%)$ were having experience in High Fidelity Practice.

The emerged themes of Perception of Undergraduate Nursing students on high-fidelity. Practices were two major themes and six basic themes with underlined line: Positive Perception and Negative Perception. Students may develop either positive or negative perception. Different factors from and within the students even beyond their influence, affect the kind of perception of on high-fidelity practices. Positive perception is the development of right skills and confidence needed to support one course of learning, chance to learn from error, through this kind of a mindset, individuals stay positive even when making errors in order to learn and develop requisite skills. Another positive perception was link between theoretic and practical learning. Negative perception comes from the feeling of inadequacy of cost of tools, lack opportunities to apply and differences between simulator and real patient, students with negative perception will develop the belief that they are not able or up to task with a given responsibility. Negative perception affects the learning ability because students tends to resistive of change. However, these factors from the environment will come impact a student's perception negatively. On positive perception, individuals developing the feeling to change their situation realize many gains. See figure 1 below: 


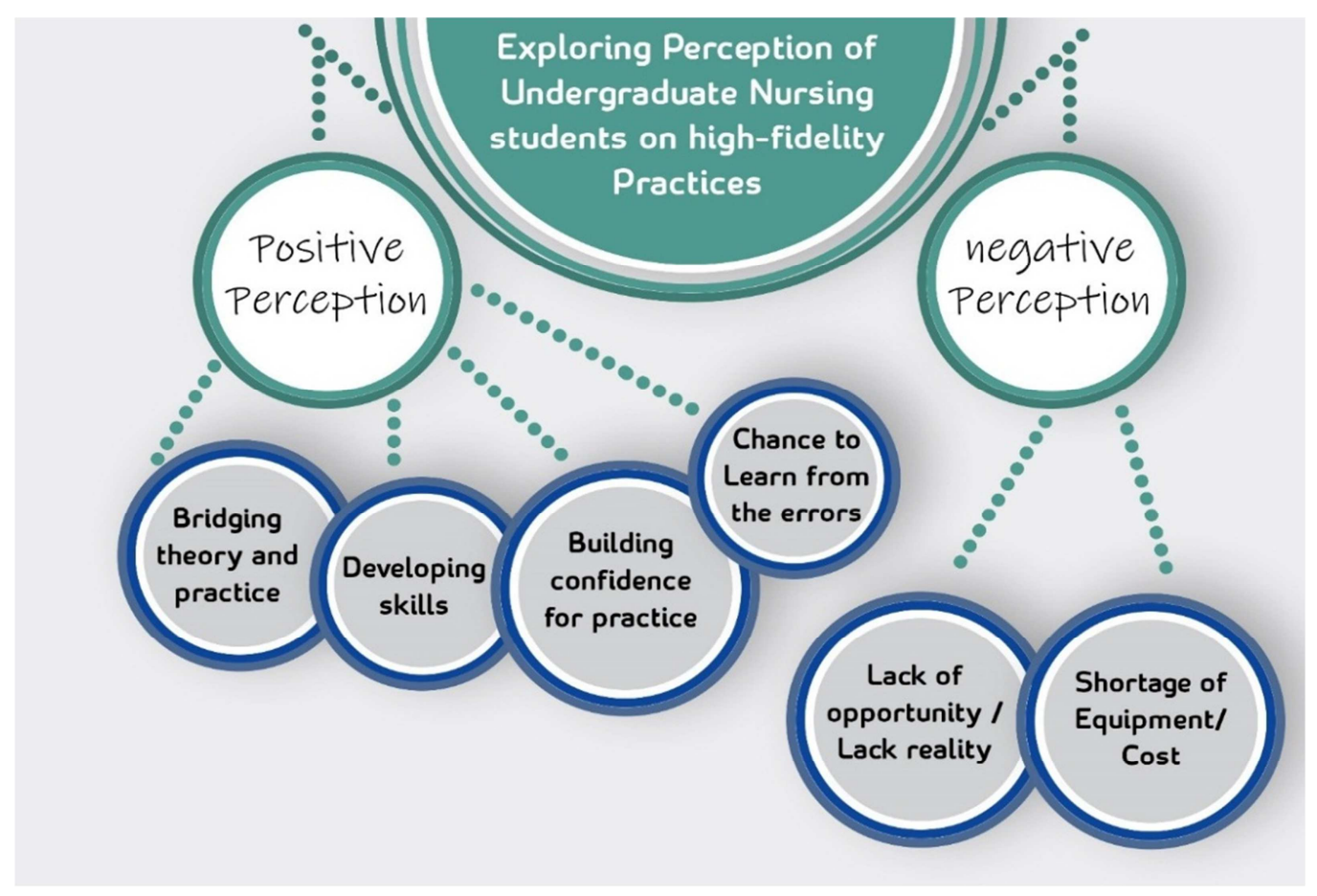

Figure 1. Framework Exploring Nursing Students Perception on high-fidelity Practices. a phenomenological study.

Figure 1 indicated the analysis of data focused on explored a particular perception of undergraduate nurses on high fidelity practices. The framework developed to set of data in which on one side, there were those that affect the perception positively while on the other hand, some influenced the process negatively. Students developed positive perception when subjected to aspects of developing skills, an opportunity to learn from the errors building their confidence as well as linked between theoretic and practice. Students who lack opportunities and or exposed to shortage of equipment became very negative.

Figure 2 showed the process of data analysis conducted to conclude the Positive Perception Theme (Theme 1). The 28 inductive codes resulted in four basic themes based on the transcripts of the interview and these became the central thoughts from the combination of the themes.

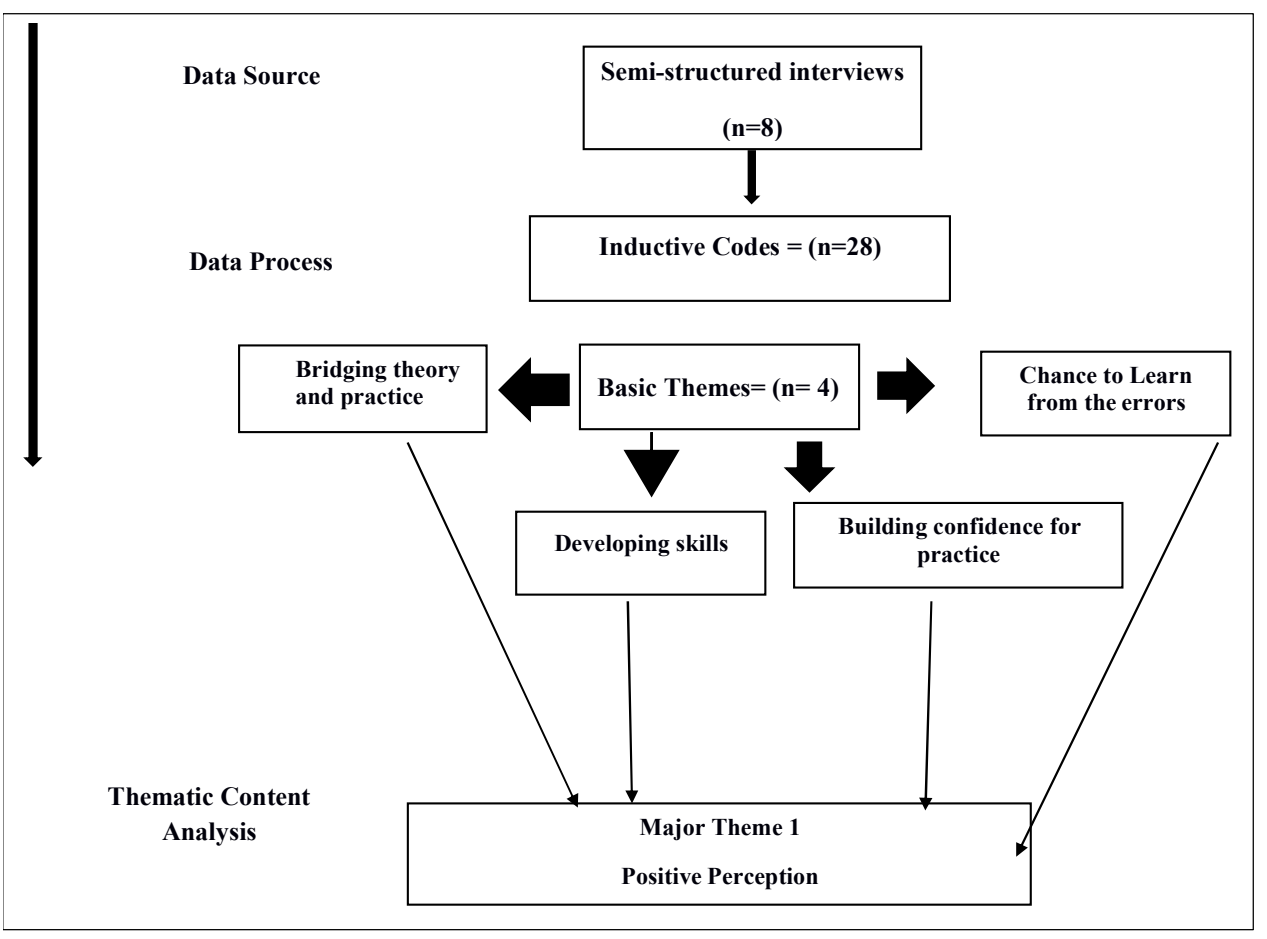

Figure 2. Process of Data Analysis in the Positive Perception (Theme 1). 


\subsection{Bridging Theory and Practice}

HFS appears to link both theory and practice. It can be used to facilitate students to apply the theoretical skills for practicing in a realistic and safe environment.

So, how did you experience this association?

Student 1:

"I think it strengthened my thinking pattern. It enabled me to move the things much faster, apply better and ensure safety to the patients as well, in the practice settings. In the absence of these simulators, we could have lacked a solid support."

Another student added that the simulation experiment helped her to assess the patient's condition in the hospital by focusing on what she studied.

Student 2:

"I was able to assess the patient situation much in advance by applying the simulator. It had given me a solid focus on the basic concept of nursing related experiences with high fidelity."

As well as some students said that the simulation experience made it linked between the study and what is applied in practice.

Student 5:

"I have gained some connection between theory and practice."

Student 7:

"It had a possible impact on my educational level as it helped me in linking the theoretical and practical information. Also to utilize my theoretical information to practice."

And the other student said that the simulation helped her remember the theoretical information that she had studied.

Student 8:

"There is an opportunity to learn in the presence of simulation from your mistakes as well as teaching more than once and help me remember the information."

\subsection{Developing Skills}

Competent skills come from the continuous process of interacting with relevant technologies and students in the simulation lab. In the process of developing skills, one also needs to interact with the simulators to help structure the operation within the area of work. In the process of developing skills, they will be able to deal with real patient later and improve communication skill which it allows for people to express themselves and also seek advice and guidance.

Student 1:

"I was told about a patient case related to respiratory syndrome by our mentor. I have gone through the concept of simulation and the related guidelines which helped me to understand the case well with the use of simulator."

Also she added the simulation experience helped her in preparing for work in the hospital through what is explained by the cases scenario in the lab.

"The clinical scenario provides the context for the simulation to build the course content and facilitate the work in hospitals."

Student 2:

"I discussed about the simulator with my nurse peers. The discussion went on to understanding the terminology related to simulation. Very soon, my concerns and knowledge gaps were addressed."

Student 5:

"I took advantage of the last terms where I learned the basics."

Student 6:

"I took advantage to learn how to give a needle and training."

Student 8:

"It is very important for application because it easy to memorize and retrieve the information."

\subsection{Building Confidence for Practice}

Confidence comes from continuous practical involvement in the simulation practice activities. There is need to appreciate the strength and weakness in order to boost the confidence for practicing.

One of them said the simulation practice gave her the confidence to do whatever the patient needed in the hospital and she was able to overcome her fears.

Student 2:

"It gave me confidence in I work these procedures without fear."

Another said.

Student 3:

"I have developed faith and motivation to deal with the complex case scenarios. I was able to feel the realism of patient case in my presence."

Also other student said.

Student 6:

"I felt the patient's pulse in more reality. This had really given me ultimate confidence."

The other said it gave her confidence in dealing with the patient.

Student 7:

"It gave me More confidence during dealing with patient, do procedures, lifting the patient, it gave us more confidence."

Student 8:

"It gives a greater confidence to the students."

Also she added.

"And gave me a preparation to clinical practice before attending and training you in the hospital."

\subsection{Chance to Learn from the Errors}

The best and the most appropriate way of learning is through trial and errors. The ability to make a trial that results in an error is essential in that it build the confidence and skills in performing a given action. Errors help student to assess the extent of their skills and the weaknesses they need to work on.

As student 3 said, she was able to distinguish between mistakes and correct work before making mistakes in dealing 
with the real patient

Student 3:

"I was able to visualize good and bad. I realized that I should overcome the mistakes which I was thinking about dealing with an real patient."

Student 4:

"I improved my ability in judging after much reflection from the guidelines of simulation and loads of high fidelity practice scenario examples encountered during."

Another said that simulations are a safe way to overcome mistakes and reduce the stress associated with working with a real patient.
Student 7:

"As well as reduces errors, reduces tension and fear for me and it a save way in dealing with simulation."

And another student added.

Student 8:

"There is no fear when applying to doll and Reduce risk and give more trust in your self."

Figure 3 indicated how data analysis was conducted to derive the Negative Perception Theme (Theme 2). The 19 inductive codes resulted in two basic themes based on the transcripts of the interviews and were later categorized as the Negative Perception or major theme.

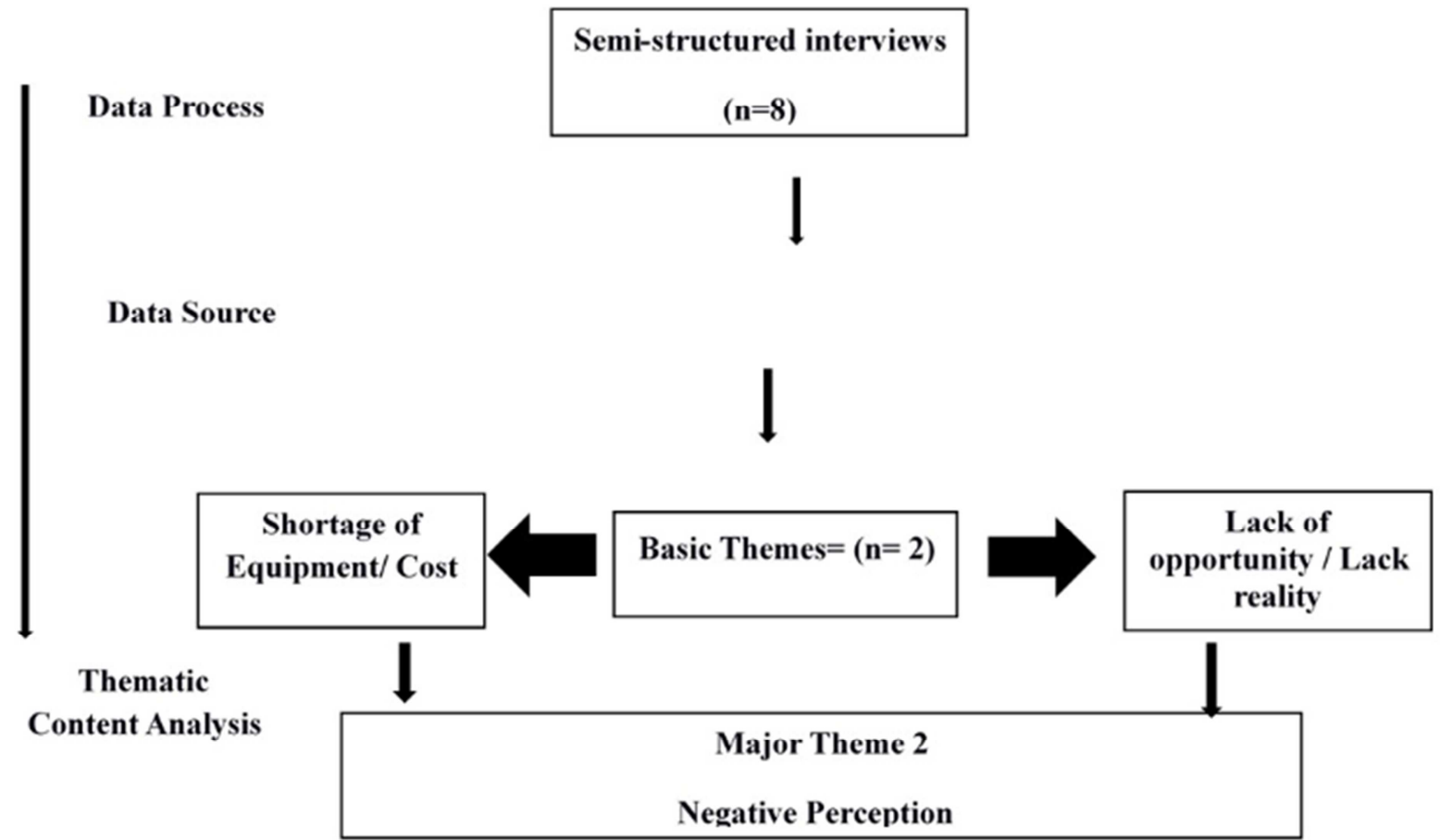

Figure 3. Process of Data Analysis in the Negative Perception Theme (2).

\subsection{Lack of Opportunity and Lack of Reality}

Developing skills and experience comes from the opportunity to try. Lack of opportunity would mean that one lacks the proper understanding of their strength and weaknesses. Lack of reality of simulators was also different simulations from the real patient such as loss of response and reflexes may effect on the ability of student to deal seriously with simulator.

Student 1:

"The big differences between simulator and the real environment of work."

Among the challenges facing the students in the simulation, where one of them said that the large number of students impede the application of all students.

Student 3:

"The teacher applies individually or with one student and not all girls apply in the lab. The number of students is large."

Another student added.

Student 5:
"There is a great difference between the work and lab environment. There isn't enough time spent in lab only 3 or 4 hours a week and in some subjects. The number of students is very high and sometimes there is no opportunity to apply."

Student 6:

"The number of students is greater than the number of equipment so there is a lack of practical application."

One of them also said that there were slight differences between the dolls in simulation and the real patient.

Student 7:

"There is a slight difference between simulation and the real patient where in the real patient there are reflexes, response, interaction, these are not found at doll. The number of students is great for teacher and there is no time for application for all students in advanced levels such as VII or VIII."

Another said.

Student 8:

"The simulation is not available according to the schedule of students. Also the time is not enough to apply all the students in each procedure." 


\subsection{Shortage of Equipment and Cost}

Lack of adequate equipment is costly affairs to students because it denies them the opportunity to deliver the appropriately. Such deficiency would also mean that the developing skills become a tedious process. Students were afraid to do any mistake that will later to discharge their practice mistake.

Student 1:

"The devices are different from real ones and are not fully available or not working. The cost of the appliances is very high so that I afraid of their damage."

Student 4:

"Everything is available in some subjects and some isn't available in others. There are not enough tools. The materials are very expensive."

Also, the size of the lab was a very large plus the size of simulator difficult to move or deal with it and some tools are not available and this is difficult to them to apply in some cases.

As another added.

Student 7

"The size of the simulators was very large and therefore I have difficulty moving it or dealing with it."

Student 8:

"Some tools are not available for example if suction Catheter not available for each student."

\section{Discussion}

In health, advances in technology are progressively clear. In nursing, HFS, as an educating and learning methodology, empowers students to gain a proficient aptitude speedier and perform better when contrasted. In this investigation, understudy's observation uncovered criticalness of HFS practically speaking, understudy connection with the HFS, association with peers in the reproduction, and future desires after high-devotion simulation. The consideration of HFS in nursing training gives large amounts of fulfillment to students with conceivable advantages in enhancing clinical abilities to use in the healing center setting. Students think about reproduction as a positive affair because of its authenticity, the profundity of encounters and the likelihood of coming into contact with circumstances which is remarkable in the genuine setting. A few investigations reported that HFS appears to make more energy than low-devotion reproduction, with benefits for students' learning [7].

In this examination, students give off an impression of being more eager, intrigued and occupied with their learning. A few investigations demonstrated that contribution in learning depends not just on the psychological capacities of students, but at the same time is impacted by motivations. The authenticity of the situations, their environment, and the way that they endeavor to comprehend extraordinary circumstances makes students extremely on the edge. Be that as it may, they consider that all the weight experienced in the simulated condition is sure in helping them to control genuine worry, as dynamic components in the adjustment and recuperation of the wellbeing of patients. Instructors assume a critical part in this procedure of clearing up with the students that everybody can commit errors and it is typical to blunder. As such, the arrangement for the simulation and the methodological thoroughness of this technology is critical [7].

The learning process entails proper institution and preparation of the mindset. It is the perception that will determine the extent to which individuals will develop requisite skills for their work. Negative perception is harmful in any learning process. It may result from a factor beyond the control of a student such as short of equipment and cost. Lack of opportunity will deprive one of the senses of reality and experience to put their skills to practice. Moreover, the effects of such negative perception will make them frustrated and unable to learn from their errors.

\section{Limitations}

This trial is falling to non-probability sampling which affect the generalizability. So, it needs to be replicated with a quantitative or mixed method study which includes randomized and larger number of participants. Likewise, further research must focus on determining the perception differences between males and females with regard to the use of simulators.

\section{Recommendation}

Students must be motivated to develop a close collaboration with their teachers and peers for acquiring maximum benefit from the simulation research. Another study to be conducted in multiple universities in different cities to assess the relationship and the differences between the student's perceptions.

\section{Conclusion}

HFS is progressively a reality in nursing schools. University students' attempt to understand the HFS technology is a professional initiative. This approach has implications for many nursing undergraduates as they need to understand the real encounters of complex clinical situations. So, instructing the HFS to students by the instructors and comprehend its impact in their preparation is mandatory in the academic curriculum. This study recognized a distinctive impression of students after encountering reasonable situations in a controlled domain, its significance to the foundation of relations and common development and its importance for their future as students and future experts. Perceiving that these reproduced clinical encounters apply diverse effects on students in building their learning, it is important to create more proof concentrated on various settings and at various levels of instruction. 


\section{References}

[1] Aebersold, M., \& Tschannen, D. (2013) "Simulation in Nursing Practice: The Impact on Patient Care" OJIN, 18 (2), Manuscript 6.

[2] Wotton, K., Davis, J., Button, D., \& Kelton, M. (2010). Third-year undergraduate nursing students' perceptions of high-fidelity simulation. J Nurs Educ, 49 (11), 632-9.

[3] Reid-Searl, K., Happell, B., Vieth, L., \& Eaton, A. (2012). High fidelity patient silicone simulation: a qualitative evaluation of nursing students' experiences. Collegian, 19 (2), 77-83.

[4] Au, M. L., Lo, M. S., Cheong, W., Wang, S. C., \& Van, I. K. (2015). Nursing students' perception of high-fidelity simulation activity instead of clinical placement: A qualitative study. Nurse Educ Today, 39, 16-21.

[5] Haraldseid, C., Friberg, F., \& Aase, K. (2015). Nursing students' perceptions of factors influencing their learning environment in a clinical skills laboratory: A qualitative study. Nurse Educ Today, 35 (9), e1-6.

[6] Ha, E. H. (2016). Undergraduate nursing students' subjective attitudes to curriculum for Simulation-based objective structured clinical examination. Nurse Educ Today, 36, 11-7.

[7] Baptista, R. C., Paiva, L. A., Gonçalves, R. F., Oliveira, L. M., Pereira, M. F., \& Martins, J. C. (2016). Satisfaction and gains perceived by nursing students with medium and high-fidelity simulation: A randomized controlled trial. Nurse Educ Today, $46,127-132$.
[8] Manchak et al (2014) Manchak, S. M., Skeem, J. L., Kennealy, P. J., \& Louden, J. E. (2014) High-fidelity specialty mental health probation improves officer practices, treatment access, and rule compliance. Law Hum Behav, 38 (5), 450-61.

[9] Littlewood, K. E. (2011). High fidelity simulation as a research tool. Best Pract Res Clin Anaesthesiol, 25 (4), 473-87.

[10] Garrett, B. M., MacPhee, M., \& Jackson, C. (2011). Implementing high-fidelity simulation in Canada: reflections on 3 years of practice. Nurse Educ Today, 31 (7), 671-6.

[11] Breymier, T., \& Rutherford-Hemming, T. (2017). Use of High-Fidelity Simulation to Increase Knowledge and Skills in Caring for Patients Receiving Blood Products. Crit Care Nurs Clin North Am, 29 (3), 369-375.

[12] Crossman, A. (2017). An Overview of Qualitative Research Methods. Retrieved from https://www.thoughtco.com/qualitative-research-methods-302 6555.

[13] NVIVO. (n. $\quad$ d.). $\quad$ Retrieved from https://goodmenproject.com/featured-content/eight-reasons-m en-need-to-deal-with-female-rejection-better-hesaid/.

[14] Anderson, R. (1997). Retrieved from http://rosemarieanderson.com/wp-content/uploads/2014/08/Th ematicContentAnalysis.pdf. 